\title{
(Hemi)Continuity of Additive Preference Preorders
}

\author{
Georgios Gerasimou \\ School of Economics 83 Finance, University of St Andrews, KY16 9AL, St Andrews, UK
}

\begin{abstract}
It is shown that the two common notions of topological continuity for preference preorders, which require closed contour sets and a closed graph respectively, are equivalent even when completeness is not assumed, provided that the domain is a normed linear space or a topological group and the preorder is additive.
\end{abstract}

Keywords: Incompleteness, continuity, hemicontinuity, additivity, independence, homotheticity

JEL: C65, D01, D11,

\section{Introduction}

In all theoretical work in economics where the aim is to provide a continuous utility, weak utility or multi-utility representation of a preference preorder, it is of interest to ensure that the topology on the preference domain is in a natural sense compatible with the preorder. This can be achieved by assuming that the latter is either continuous, in the sense that

Email address: gg26@st-andrews.ac.uk (Georgios Gerasimou) 
it is closed as a subset of the product space, or hemicontinuous, in the sense that the upper and lower contour sets of the preorder are closed for every element of the domain.

It is well-known that if the preorder is complete so that any two elements are preference-comparable, then continuity and hemicontinuity are equivalent (Ward, 1954; Bridges and Mehta, 1995). When the preorder is not complete, however, continuity is generally stronger than hemicontinuity. A general characterization of the additional structure that continuity imposes in this more general setting is provided in Gerasimou (2013). However, it seems to be unknown at present whether mild conditions on the preference relation and/or on its domain suffice for the equivalence between the two topological properties to be restored in the context where completeness is not assumed.

The contribution of this paper is to show that continuity and hemicontinuity are equivalent when the domain is a normed vector space or a topological group and the preorder is additive. In the former case where the space has a linear structure, additivity is shown to be satisfied if the preorder is homothetic and also obeys the independence axiom (in fact, it is shown that additivity and independence are equivalent under a weak notion of homotheticity). Despite the well-known descriptive shortcomings of these axioms, they are all essential, for instance, in modelling individuals who maximize subjective expected value in the sense of de Finetti $(1937)^{1}$, even when completeness is not assumed (see Ghirardato et al. 2004).

With regard to the relevant literature, two recent papers on the problem of identifying the way in which different notions of preference continuity are

\footnotetext{
${ }^{1}$ See also Chapter 10 in Gilboa (2009).
} 
logically related and whether they become equivalent under certain conditions are Karni (2007) and Gilboa et al. (2010). Karni (2007) studied the relationship between Archimedean and mixture continuity for a complete preorder that is defined on a probability simplex. He found a condition, called "local mixture dominance", which, jointly with Archimedean continuity, characterizes mixture continuity. Moreover, in the context of preferences over Anscombe-Aumann acts, Gilboa et al (2010, Lemma 3) proved that a possibly incomplete preorder that satisfies monotonicity and independence is continuous in the above sense if and only if it is mixture-continuous, provided a technical domain restriction is satisfied.

\section{Preliminaries}

A preordered topological space $(X, \tau, \succsim)$ consists of a set $X$, a topology $\tau$ and a reflexive and transitive relation $\succsim$ on $X$. I will write $(X, \succsim)$ or simply $X$ and refer to it as a preordered space. If the sets $U(x):=\{y \in X: y \succsim x\}$ and $L(x):=\{y \in X: x \succsim y\}$ are closed for some $x \in X$, then the preorder is upper-and lower-hemicontinuous at $x$, respectively. A preordered space $X$ is hemicontinuously preordered if the sets $U(x)$ and $L(x)$ are closed for all $x \in X$. It is continuously preordered if $\succsim$ is closed as a subset of the product space $X \times X$. The complement of a preorder $\succsim$ in $X \times X$ is denoted by $\nsucceq$. The complement of a set $A \subset X$ is denoted by $A^{c}$.

The first example below shows a preorder that is hemicontinuous but not continuous. It was suggested to me by Ettore Minguzzi (Florence).

Example 1. Let $X=\mathbb{R}$ with its natural topology. Define the relation $\succsim$ on 
$X$ by

$$
x \succsim y \Longleftrightarrow\left\{\begin{array}{l}
x=y \\
\text { or } \\
y<-1 \text { and } x=-y
\end{array}\right.
$$

The relation $\succsim$ is clearly reflexive and antisymmetric. It is also trivially transitive (if $x \succ y$, then $y \nsucc z$ for all $z \in X$ ). Hence, it is a partial order. By definition, $U(x)=\{x,-x\}$ for all $x<-1$ and $U(x)=\{x\}$ for all $x \geq-1$. Moreover, $L(x)=\{x\}$ for all $x \leq 1$ and $L(x)=\{x,-x\}$ for all $x>1$. Thus, $\succsim$ is hemicontinuous. Now define the sequences $\left(x_{n}\right),\left(y_{n}\right)$ in $X$ by $x_{n}=1+\frac{1}{n}$ and $y_{n}=-1-\frac{1}{n}$. It holds that $x_{n} \succsim y_{n}$ for all $n \in \mathbb{N}$, $x_{n} \rightarrow x=1, y_{n} \rightarrow y=-1$ and $x \succsim y$. Hence, $\succsim$ is not continuous.

The next example features a preorder on a probability simplex which also fails to be continuous despite being hemicontinuous.

Example 2. Let $X=\left\{\left(p^{1}, p^{2}, p^{3}\right) \in \mathbb{R}_{+}^{3}: p^{1}+p^{2}+p^{3}=1\right\}$ with the induced topology. For $p=\left(p^{1}, p^{2}, p^{3}\right) \in X$, let $p^{\prime} \in X$ be defined by $p^{\prime}=\left(p^{3}, p^{2}, p^{1}\right)$. Define the relation $\succsim$ on $X$ by

$$
p \succsim q \Longleftrightarrow\left\{\begin{array}{l}
p=q \\
o r \\
q=p^{\prime} \text { and } p^{3}>\frac{2}{3}
\end{array}\right.
$$

This relation is clearly reflexive. Moreover, if $p \succsim q$ and $p \neq q$, then $q=p^{\prime}$ holds by construction, and there is no $r \in X$ such that $q \succsim r$ and $q \neq r$. Indeed, suppose the latter is not true. Then, $q^{3}>\frac{2}{3}$ and $r=q^{\prime}=p$, because $q=p^{\prime}$ implies $q^{\prime}=p$. But since $q^{\prime}=p \in X, q^{3}>\frac{2}{3}$ implies $q^{\prime 3}=p^{3}<\frac{2}{3}$. This is a contradiction. Therefore, $\succsim$ is trivially transitive. Finally, the 
previous argument also establishes that $\succsim$ is antisymmetric, and hence a partial order.

From the above definition of $\succsim$ we have that, for all $q \in X, U(q)=\{q\}$ if $q^{1} \leq \frac{2}{3}, U(q)=\left\{q, q^{\prime}\right\}$ if $q^{1}>\frac{2}{3}, L(q)=\{q\}$ if $q^{3} \leq \frac{2}{3}$ and $L(q)=$ $\left\{q, q^{\prime}\right\}$ if $q^{3}>\frac{2}{3}$. These sets are closed for all $q \in X$ and therefore $\succsim$ is hemicontinuous. Let $\left(p_{n}\right),\left(q_{n}\right)$ be sequences in $X$ defined by

$$
\begin{aligned}
& p_{n}=\left(\frac{1}{6}-\frac{1}{6 n}, \frac{1}{6}, \frac{2}{3}+\frac{1}{6 n}\right) \\
& q_{n}=\left(\frac{2}{3}+\frac{1}{6 n}, \frac{1}{6}, \frac{1}{6}-\frac{1}{6 n}\right)
\end{aligned}
$$

Clearly, $p_{n} \succsim q_{n}$ for all $n \in \mathbb{N}, p_{n} \rightarrow\left(\frac{1}{6}, \frac{1}{6}, \frac{2}{3}\right), q_{n} \rightarrow\left(\frac{2}{3}, \frac{1}{6}, \frac{1}{6}\right)$, and $p \nsucceq q$. Thus, $\succsim$ is not continuous.

\section{Main Result}

If the preference domain $X$ is a vector space, then a preorder $\succsim$ on $X$ is additive if $x \succsim y$ implies $x+z \succsim y+z$ for all $z \in X$. The behavioural implications (particularly in relation to risk neutrality) of additivity in the context of choice under uncertainty are discussed in detail in Gilboa (2009).

The paper's main result is the following:

Theorem 1. Suppose $(X, \succsim)$ is a preordered normed vector space and $\succsim i s$ additive. The following are equivalent.

(a) $\succsim$ is upper-or lower-hemicontinuous at 0 .

(b) $\succsim$ is hemicontinuous.

(c) $\succsim$ is continuous.

Proof. It is obvious that (c) implies (a). It will be shown that (a) implies (b) implies (c). 
(a) $\Rightarrow$ (b). Without loss of generality, assume that $\succsim$ is upper-hemicontinuous at 0 . Suppose $\left(y_{n}\right)$ is a sequence in $X$ such that $0 \succsim y_{n}$ for all $n \in \mathbb{N}$. Since $X$ is a vector space and $y_{n} \in X$, it follows that $-y_{n} \in X$. Since $\succsim$ is additive, $0 \succsim y_{n}$ is equivalent to $-y_{n} \succsim 0$ for all $n$. Suppose $y_{n} \rightarrow y$. Since $U(0)$ is closed and $-y_{n} \rightarrow-y$ it follows that $-y \succsim 0$, or $0 \succsim y$. Hence, $L(0)$ is also closed.

Now consider some arbitrary $x \in X$. Suppose $\left(y_{n}\right)$ is a sequence satisfying $y_{n} \succsim x$ for all $n$, and let $y_{n} \rightarrow y$. It holds that $y_{n}-x \succsim 0$ for all $n$. Since $y_{n}-x \rightarrow y-x$, it follows from the above that $y-x \succsim 0$ or, equivalently, $y \succsim x$. Thus $U(x)$ is closed. A symmetric argument shows that $L(x)$ is closed too.

(b) $\Rightarrow(\mathrm{c})$. Suppose $X$ is normed by $\|\cdot\|$ and let the topology on $X$ be generated by the metric $d(\cdot, \cdot)$ that is induced by this norm. Suppose $x \nsucceq y$. From hemicontinuity, the sets $L(x)^{c}$ and $U(y)^{c}$ are open. Hence, $x \nsucceq y$ implies there are open balls $B_{\epsilon_{x}}(x)$ and $B_{\epsilon_{y}}(y)$ such that $x^{\prime} \nsucceq y$ and $x \not y^{\prime}$ for all $x^{\prime} \in B_{\epsilon_{x}}(x)$ and all $y^{\prime} \in B_{\epsilon_{y}}(y)$, respectively. Define $\epsilon:=\min \left\{\epsilon_{x}, \epsilon_{y}\right\}$. It holds that

$$
x \nsucceq y^{\prime} \quad \text { and } \quad x^{\prime} \nsucceq y \quad \forall x^{\prime} \in B_{\epsilon}(x), y^{\prime} \in B_{\epsilon}(y)
$$

Now consider the distance $\frac{\epsilon}{2}$ and suppose, per contra, that $B_{\frac{\epsilon}{2}}(x) \times B_{\frac{\epsilon}{2}}(y)$ $\not \subset \nsucceq$. Then, there exist $x^{\prime} \in B_{\frac{\epsilon}{2}}(x)$ and $y^{\prime} \in B_{\frac{\epsilon}{2}}(y)$ such that $x^{\prime} \succsim y^{\prime}$. Let $v:=x-x^{\prime}$. By assumption, $v \in X$. Moreover, since $\succsim$ is additive, it follows that

$$
\begin{aligned}
x^{\prime} \succsim y^{\prime} & \Longrightarrow x^{\prime}+v \succsim y^{\prime}+v \\
& \Longrightarrow x \succsim y^{\prime}+x-x^{\prime} .
\end{aligned}
$$


From the triangle inequality we get

$$
d\left(y, y^{\prime}+x-x^{\prime}\right)=\left\|y-y^{\prime}-x+x^{\prime}\right\| \leq\left\|y-y^{\prime}\right\|+\left\|x^{\prime}-x\right\|<\frac{\epsilon}{2}+\frac{\epsilon}{2}=\epsilon .
$$

It follows from (3) that $y^{\prime}+x-x^{\prime} \in B_{\epsilon}(y)$ and therefore, from (1), that $x \nsucceq y^{\prime}+x-x^{\prime}$. But this contradicts (2). Therefore, $x \nsucceq y$ implies that an open neighborhood of $(x, y)$ can be found that is contained in $\nsucceq$. Hence, $\nsucceq$ has an open graph, or, equivalently, $\succsim$ is continuous.

Remark 1. The Euclidean space $\mathbb{R}^{n}$ with the usual partial ordering $\geq$ is an example of a normed vector space with an additive hemicontinuous preorder. As is well-known, $\geq$ is also continuous. On the other hand, the hemicontinuous but not continuous partial order in Example 1 is defined on a normed vector space but fails to be additive (e.g. $2 \succsim-2$ but $2+1=3 \not-1=-2+1)$, whereas the one in Example 2 is not defined on a normed vector space and, by construction, is not additive either.

The logical relationship between additivity and some other well-known preference axioms is studied next. Recall first that a preorder $\succsim$ on $X$ is affine if $x \succsim y$ implies $\alpha x+(1-\alpha) z \succsim \alpha y+(1-\alpha) z$ for all $\alpha \in[0,1]$ and all $z \in X$, and homothetic if $x \succsim y$ implies $\alpha x \succsim \alpha y$ for all $\alpha>0$. I will refer to $\succsim$ as lower-homothetic if $x \succsim y$ implies $\alpha x \succsim \alpha y$ for all $\alpha \in(0,1)$.

Claim 2. A lower-homothetic preorder $\succsim$ on a vector space $X$ is affine if and only if it is additive.

Proof. Assume first that $\succsim$ is lower-homothetic and affine, and suppose $x \succsim y$. It holds that $\alpha x+(1-\alpha) z \succsim \alpha y+(1-\alpha) z$ for all $\alpha \in(0,1)$ and all $z \in X$. Since $\succsim$ is lower-homothetic, $\frac{\alpha}{1-\alpha} x+z \succsim \frac{\alpha}{1-\alpha} y+z$. When $\alpha=\frac{1}{2}$, this is equivalent to $x+z \succsim y+z$. 
Conversely, assume that $\succsim$ is lower-homothetic and additive, and let $x \succsim y$. Consider $\alpha \in(0,1)$. From lower-homotheticity, $\alpha x \succsim \alpha y$. From additivity, $\alpha x+(1-\alpha) z \succsim \alpha y+(1-\alpha) z$.

Remark 2. Consider a convex cone $C$ in a topological vector space $X$, i.e. a convex subset of $X$ with the property that $x \in C$ implies $\lambda x \in C$ for all $\lambda \geq 0$. The cone $C$ induces a preorder $\succsim$ on $X$ by $x \succsim y$ if and only if $x-y \in C$. Here, $C$ coincides with the upper-contour set $U(0)$ of $\succsim$. It is well-known that this preorder $\succsim$ is continuous if and only if $C$ is closed (see pp. 19-20 in Wong and $\mathrm{Ng}$ (1973)). ${ }^{2}$ Theorem 1 relaxes the conditions on $\succsim$ in this result by not requiring $U(0)$ to be a convex cone, while retaining additivity. Therefore, $\succsim$ is not assumed to be convex or homothetic (and hence, in view of Claim 2, not affine either).

As already noted, a context where a possibly incomplete preference preorder satisfies the conditions of Theorem 1 (in fact, all three conditions in the statement of Claim 2) is that of subjective expected value with incomplete preferences. Such a representation is given in Proposition A.2 in Ghirardato et al. (2004). There, the agent is portrayed as having incomplete preferences over monetary bets as well as a set of priors over the states of the world, and to weakly prefer one bet over another if and only if it yields a weakly higher expected value according to each prior (see also Theorem 1 in Bewley (2002) for a strict-preference analogue of this result). Although full continuity was assumed directly in Ghirardato et al. (2004), in light of Theorem 1 this can be replaced by the weaker notion of hemicontinuity

\footnotetext{
${ }^{2}$ I thank a reviewer for this reference.
} 
or even upper- or lower-hemicontinuity at the origin, at least whenever the domain of acts is a linear space.

Finally, as is well-known since Schmeidler (1971) and, more recently, Dubra (2011), when sufficiently strong continuity notions are imposed on a preorder that is defined on some suitably rich domain, the preorder is actually complete. However, as remarked above with the example of the usual partial ordering, there also exist continuous preorders that are additive as well as convex and homothetic which are, in fact, incomplete. Therefore, the interaction of additivity and (hemi)continuity is not sufficiently strong to imply completeness.

\section{Extension to Topological Groups}

The proof of Theorem 1 that was given above did not make use of the fact that linear spaces are closed under the operation of scalar multiplication. This suggests the possibility that the essence of the result extends to topological groups, where this structure is not imposed. To this end, let $(G, \succsim)$ be a preordered topological group, with $1 \in G$ the identity element of the group. That is, $1 \in G$ is the unique element with the property that, for all $a \in G$, the equation $1 a=a 1=a$ holds.

In this context, the preorder $\succsim$ is additive if $x \succsim y$ implies $x z \succsim y z$ for all $x, y, z \in X$. The following extension of Theorem 1 was suggested to me by Hans-Peter Künzi (Cape Town).

Theorem 3. Suppose $(G, \succsim)$ is a preordered topological group and $\succsim$ is additive. The following are equivalent:

(a) $\succsim$ is upper-or lower-hemicontinuous at 1 . 
(b) $\succsim$ is continuous.

(c) $\succsim$ is hemicontinuous.

Proof. It is clearly true that (b) implies (c) and (c) implies (a). It will be shown that (a) implies (b). Without loss of generality, let $\succsim$ be upperhemicontinuous at 1 . Suppose that $\left(x_{d}, y_{d}\right)_{d \in D}$ is a net converging to $(x, y) \in$ $G \times G$, and that $x_{d} \succsim y_{d}$ for all $d \in D$. Since $G$ is a group, $w z^{-1} \in G$ for all $w, z \in G$. Thus, $x_{d} y_{d}^{-1} \in G$ and $x_{d} y_{d}^{-1} \succsim 1$ for all $d \in D$. Since $U(1)$ is closed and $\left(x_{d} y_{d}^{-1}\right) \rightarrow x y^{-1}$ because $G$ is a topological group, it follows that $x y^{-1} \succsim 1$, or $x \succsim y$. Thus, $\succsim$ is continuous.

With regard to some related literature on topological groups, the reader is referred to Candeal-Haro and Indurain-Eraso (1992) for a weak utility representation of a partial order on such domains.

\section{Acknowledgments}

I am grateful to Ettore Minguzzi for showing me Example 1 which uncovered an important error in a previous version of the paper. I also thank Hans-Peter Künzi for showing me that Theorem 1 can be extended to topological groups, and two referees for suggestions that led to improvements in the paper. Any errors remaining are my own.

\section{References}

Bewley, T. F., 2002. Knightian decision theory. Part I. Decisions in Economics and Finance 25, 79-110, (First version: 1986).

Bridges, D. S., Mehta, G. B., 1995. Representations of preference orderings. Lecture Notes in Economics and Mathematical Systems 422. Berlin: Springer.

Candeal-Haro, J. C., Indurain-Eraso, E., 1992. Utility functions on partially ordered topological groups. Proceedings of the American Mathematical Society 115, 765-767. 
de Finetti, B., 1937. La prévision: Ses lois logiques, ses sources subjectives. Annales de l'Institut Henri Poincaré 7, 1-68.

Dubra, J., 2011. Continuity and completeness under risk. Mathematical Social Sciences $61,80-81$.

Gerasimou, G., 2013. On continuity of incomplete preferences. Social Choice and Welfare $41,157-167$.

Ghirardato, P., Maccheroni, F., Marinacci, M., 2004. Differentiating ambiguity and ambiguity attitude. Journal of Economic Theory 118, 133-173.

Gilboa, I., 2009. Theory of Decision under Uncertainty. Econometric Society Monographs 45. Cambridge University Press.

Gilboa, I., Maccheroni, F., Marinacci, M., Schmeidler, D., 2010. Objective and subjective rationality in a multiple prior model. Econometrica 78, 755-770.

Karni, E., 2007. Archimedean and continuity. Mathematical Social Sciences 53, 332-334.

Schmeidler, D., 1971. A condition for the completeness of partial preference relations. Econometrica 39, 403-404.

Ward, L. E., 1954. Partially ordered topological spaces. Proceedings of the American Mathematical Society 5, 144-161.

Wong, Y.-C., Ng, K.-F., 1973. Partially ordered topological vector spaces. Oxford: Clarendon. 